\title{
A bounded cumulative hazard model with a change-point according to a threshold in a covariate for right-censored data
}

\begin{abstract}
In most recent clinical studies, the focus is on estimation of the proportion of patients who are cured and who will therefore never experience the event of interest again. This article investigates a survival model with cure fraction and change-point effect based on the bounded cumulative hazard model $(\mathrm{BCH})$. The maximum likelihood approach to estimate the unknown parameters is used. A major difficulty here is that the likelihood function is not differentiable with respect to a change point parameter. To address this problem a smoothed likelihood approach is proposed. Simulation studies have been conducted in this study to assess the efficiency of the estimators under various practical situations. Numerical results show the satisfying performance of the proposed estimates and that the proposed model represents a useful addition to the literature of the $\mathrm{BCH}$ model.
\end{abstract}

Keyword: BCH model; Change-point model; Smoothing; Right-censoring; Maximum likelihood estimation 